\title{
Efecto del DDGS (granos secos de destilería con solubles) sobre los parámetros productivos y la calidad de huevos en gallinas Lohmann Brown - classic
}

\section{Effect of DDGS (distillers dried grains with solubles) on performance and quality of Lohmann Brown hens eggs - classic}

\author{
Elmer Sopla Cotrina ${ }^{1},{ }^{*}$ Segundo José Zamora Huamán², Francys Mitchel Canto Sáenz ${ }^{3}$
}

\section{RESUMEN}

El objetivo fue evaluar el efecto de la inclusión de diferentes niveles de DDGS (granos secos de destilería con solubles) sobre los parámetros productivos y la calidad de huevos en gallinas Lohmann Brown - Classic. Se utilizaron 48 gallinas, distribuidas en 4 tratamientos ( $0,10,15$ y 20\% de DDGS más enzimas), con 3 repeticiones de 4 gallinas cada una. La adición de DDGS más enzimas en la dieta de gallinas ponedoras mejora el porcentaje de postura, siendo $15 \%$ de adición, el mejor con 95,2\%; el consumo de alimento, siendo el 15\% de adición, el de mejores resultados con 117,7 g/ave/día; y la conversión alimenticia siendo 15 y $20 \%$ las dosis con mejores resultados $(2,0)$. En cuanto a la calidad de huevos, con el $20 \%$ de adición se obtuvo mejores resultados $(7,5)$ y mejor peso $(63,7 \mathrm{~g})$.

Palabras clave: Gallinas ponedoras, Lohmann Brown-Classic, DDGS, enzimas, parámetros, pigmentante.

\begin{abstract}
The purpose of this work was to evaluate the effect of the inclusion of different levels of DDGS (Distiller's dried grains with solubles) on the production parameters and egg quality in Lohmann Brown - Classic hens. 48 hens were used, for which they were distributed in four treatments (0, 10, 15 and 20\% of DDGS plus enzymes), with three replicates of four hens each. Adding DDGS plus enzymes to the laying hens diet improves the percentage of laying, $15 \%$ in addition, the best with $95.2 \%$; the food consumption, the best results with $117.7 \mathrm{~g} / \mathrm{bird} / \mathrm{day}$; and feed conversion being 15 and $20 \%$ the doses with better results (2.0). As for egg quality, with additional $20 \%$ better results (7.5) and better weight $(63.7 \mathrm{~g})$ were obtained.
\end{abstract}

Keywords: Laying hens, Lohmann Brown - Classic, DDGS, enzymes, parameters.

\footnotetext{
Bachiller de la Facultad de Ingeniería Zootecnista, Agronegocios y Biotecnología de la Universidad Nacional Toribio Rodríguez de Mendoza de Amazonas.

${ }^{2}$ *Ingeniero Zootecnista. Profesor auxiliar a tiempo completo de la Facultad Ingeniería Zootecnista, Agronegocios y Biotecnología de la Universidad Nacional Toribio Rodríguez de Mendoza de Amazonas. Correo electrónico: s.zamora.fizab@untrm.edu.com

${ }^{3}$ Ingeniero Zootecnista del Proyecto PRONUT de la Universidad Nacional Toribio Rodríguez de Mendoza de Amazonas. Correo electrónico: cantosaenz@gmail.com
} 


\section{INTRODUCCIÓN}

Los DDGS (granos secos de destilería con solubles), son un subproducto de la industria del etanol a partir de la fermentación de almidones de granos de cereales. El maíz es la principal fuente de almidones para la producción de etanol en los Estados Unidos. Los DDGS como ingredientes en la alimentación de las aves, son una fuente considerable de proteína, aminoácidos, energía, fósforo y otros nutrientes, pero el principal problema de su empleo hace algunos años, eran la gran variabilidad en el contenido y su calidad. Más recientemente, DDGS de color dorado provenientes de nuevas plantas de etanol con un proceso adecuado de secado, tuvieron en promedio más proteína, grasa, calcio y fósforo; además de esto el precio y su disponibilidad hacen a los DDGS como ingredientes atractivos. (Blas, Mateos y Rebollar, 2007).

Los DDGS pueden proporcionar una cantidad significativa de energía, aminoácidos y fósforo a las dietas de aves (Spiehs et al., 2002).

Las gallinas Lohmann Brown presentan fortalezas de impacto productivo y económico, como la masa de huevo, relacionada con la alta persistencia que las caracteriza, con huevos de gran tamaño, con cáscaras de excelente calidad y pigmentación. De igual manera se destacan por su capacidad de adaptación a condiciones extremas de clima y de recuperación frente a desafíos sanitarios y por el buen peso de la gallina al final del ciclo (Paucar, 2016).

El huevo de gallina, es desde la antigüedad un alimento muy importante para el hombre y en la actualidad su consumo está casi generalizado. Un huevo está formado, básicamente, por una yema central (31\%) rodeada por el albumen o clara (58\%) $\mathrm{y}$ todo ello envuelto por una cáscara externa (11\%). El huevo tiene contenidos moderados en calorías y ácidos grasos (AG) saturados. Ofrece una proteína con un perfil en aminoácidos ideal para las necesidades del organismo, una alta proporción de $\mathrm{AG}$ insaturados, todas las vitaminas excepto la $\mathrm{C}$ y minerales esenciales de forma concentrada. Estos macro y micronutrientes están conservados y protegidos por la cáscara (Aparicio, Barroeta, López-Sobaler y Ortega, 2008).

\section{MATERIALES Y MÉTODOS}

La investigación se realizó en el módulo de aves de la Estación Experimental Chachapoyas de la Universidad Nacional Toribio Rodríguez de Mendoza de Amazonas - UNTRM.
La fase experimental tuvo una duración de 45 días, entre los meses de noviembre y diciembre del 2015.

Para formular la ración alimenticia, se utilizó un programa computarizado DAPP Nutrition, de acuerdo a los requerimientos y estado de producción de las gallinas Lohmann Brown - classic. Para determinar el contenido nutricional del DDGS se utilizó el equipo NIR en el laboratorio de Nutrición Animal y Bromatología de Alimentos de la UNTRM.

La ración empleada en todos los tratamientos se muestra en la Tabla 1

Tabla 1. Ración alimenticia formulada con inclusión de DDGS mas enzimas para gallinas Lohmann Brown - classic.

\begin{tabular}{|c|c|c|c|c|}
\hline INGREDIENTE & T1 & $\mathbf{T 2}$ & T3 & T4 \\
\hline Maíz nacional & 60,955 & 56,110 & 52,401 & 47,095 \\
\hline DDGS & 0,000 & 10,096 & 15,135 & 20,205 \\
\hline $\begin{array}{l}\text { Torta de soya } \\
44 \%\end{array}$ & 23,01 & 19,15 & 17,202 & 15,498 \\
\hline $\begin{array}{l}\text { Carbonato de } \\
\text { calcio }\end{array}$ & 8,388 & 8,489 & 8,553 & 9,015 \\
\hline Afrecho de trigo & 5,110 & 4,822 & 5,200 & 6,130 \\
\hline Aceite de soya & 0,982 & 0,452 & 0,108 & 0,059 \\
\hline Fosfato dicálcico & 0,700 & 0,579 & 0,488 & 1,212 \\
\hline Sal compun & 0,237 & 0,215 & 0,204 & 0,348 \\
\hline $\begin{array}{l}\text { Proapak } 4^{a} \\
\text { ponedor }\end{array}$ & 0,137 & 0,137 & 0,137 & 0,137 \\
\hline Metionina dl & 0,203 & 0,192 & 0,186 & 0,079 \\
\hline $\begin{array}{l}\text { Cloruro de colina } \\
60 \%\end{array}$ & 0,064 & 0,064 & 0,064 & 0,064 \\
\hline $\begin{array}{l}\text { Bicarbonato de } \\
\text { sodio }\end{array}$ & 0,055 & 0,054 & 0,054 & 0,055 \\
\hline Zinc bacitacina 10 & 0,045 & 0,045 & 0,045 & 0,045 \\
\hline $\begin{array}{l}\text { Sulfato colistina } \\
10 \%\end{array}$ & 0,050 & 0,050 & 0,050 & 0,050 \\
\hline $\begin{array}{l}\text { Econase xt postu } \\
75 j\end{array}$ & 0,009 & 0,010 & 0,013 & 0,032 \\
\hline $\begin{array}{l}\text { Quantum blue } \\
\text { post } 8\end{array}$ & 0,009 & 0,009 & 0,009 & 0,009 \\
\hline Lisina & 0,045 & 0,121 & 0,150 & 0,110 \\
\hline Totales & 100 & 100 & 100 & 100 \\
\hline Costos (S/,) & 1,62 & 1,61 & 1,60 & 1,59 \\
\hline
\end{tabular}

La ración alimenticia por tratamientos, se llevó a un molino donde se mezcló todos los ingredientes para luego alimentar a las gallinas. Posteriormente, se evaluó los parámetros productivos y la calidad de huevos.

En la investigación se utilizó un Diseño Completamente al Azar (DCA), con cuatro tratamientos, cuyo único factor fue el contenido de DDGS, tal como se observa en la Tabla 2. 
Tabla 2. Niveles que intervienen en el estudio de la investigación (DCA)

\begin{tabular}{lll}
\hline Tratamiento & \multicolumn{2}{l}{ Descripción } \\
\hline T1 & Dieta con $0 \%$ de DDGS + enzimas \\
\hline T2 & Dieta con $10 \%$ de DDGS + \\
& enzimas & \\
\hline T3 & Dieta con $15 \%$ de DDGS + \\
& enzimas \\
\hline T4 & Dieta con $20 \%$ de DDGS + \\
& enzimas & \\
&
\end{tabular}

Para la apariencia, el sabor y olor de los huevos, se empleó trabajó con un panel de 10 personas (alumnos egresados de la Carrera Profesional de Ingeniería Zootecnista), para lo cual se empleó una escala tipo Likert de 1 a 4, donde: (1) malo, (2) regular, (3) bueno y(4) excelente.

\section{RESULTADOS}

En la Figura 1 se muestra los porcentajes de postura de gallinas de 49 semanas de edad, que recibieron DDGS con enzimas en la dieta.

\section{Efecto de los tratamientos sobre el $\%$ de postura}

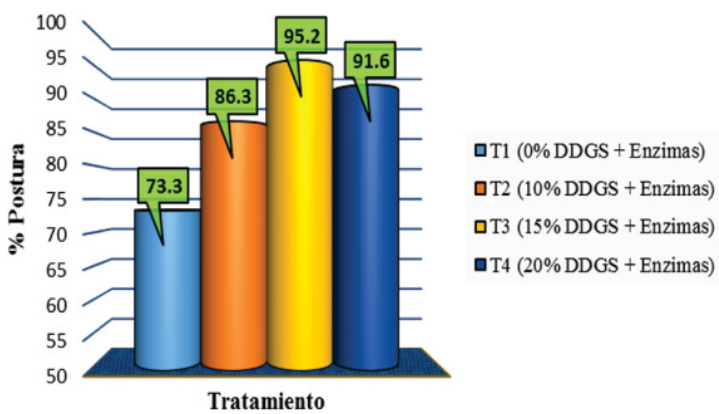

Figura 1. Porcentaje de postura logrado pos los tratamientos

Todos los tratamientos superaron al testigo y son diferentes a éste (Tabla 3 ) y los valores más altos se obtuvieron con 15 y $20 \%$ de DDGS con enzimas.
Tabla 3. Comparación de Medias Tukey de porcentaje de postura

\begin{tabular}{|l|l|}
\hline Tratamiento & \% de postura (media) \\
\hline T1 & $95,2 \pm 17,33 \mathrm{a}$ \\
\hline T2 & $91,6 \pm 17,33 \mathrm{a}$ \\
\hline T3 & $86,3 \pm 17,33 \mathrm{a}$ \\
\hline T4 & $73,3 \pm 17,33 \mathrm{~b}$ \\
\hline
\end{tabular}

En la Figura 2 se presenta los datos de consumo de alimento registrados en la investigación.

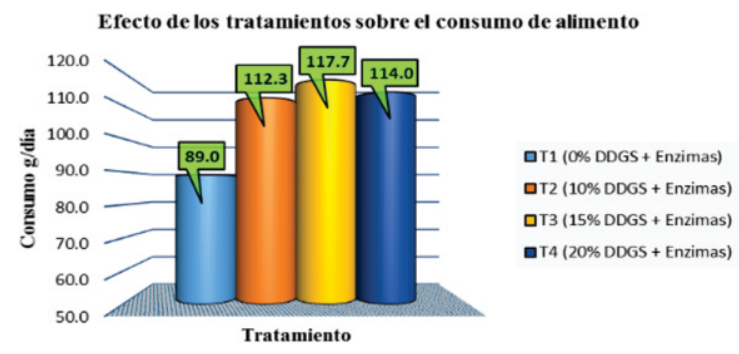

Figura 2. Consumo de alimento para todos los tratamientos

El T3 (15\% DDGS) mostró mejor resultado en consumo de alimento, con 117,7 g de alimento/ave/día y el T1 (0\% DDGS) mostró el menor consumo con $89,0 \mathrm{~g}$ de alimento/ave/día.

Tabla 4. Comparación de Medias Tukey de consumo de alimento

\begin{tabular}{|l|c|}
\hline Tratamiento & $\begin{array}{l}\text { Consumo de alimento } \\
\text { (media g) }\end{array}$ \\
\hline T1 & $117,7 \pm 7,09 \mathrm{a}$ \\
\hline T2 & $114,4 \pm 7,09 \mathrm{a}$ \\
\hline T3 & $112,3 \pm 7,09 \mathrm{a}$ \\
\hline T4 & $89,0 \pm 7,09 \mathrm{~b}$ \\
\hline
\end{tabular}

En la Tabla 4, se observa que los tratamientos T2, T3 y T4 presentan diferencias significativas con respecto al T1.

En la Figura 3, se observa que el tratamiento testigo y el T2 (10\% DDGS) reportaron la misma conversión alimenticia de $2,07 \mathrm{~kg}$, el T3 (15\% DDGS) y T4 (20\% DDGS) con 2,00 kg. 


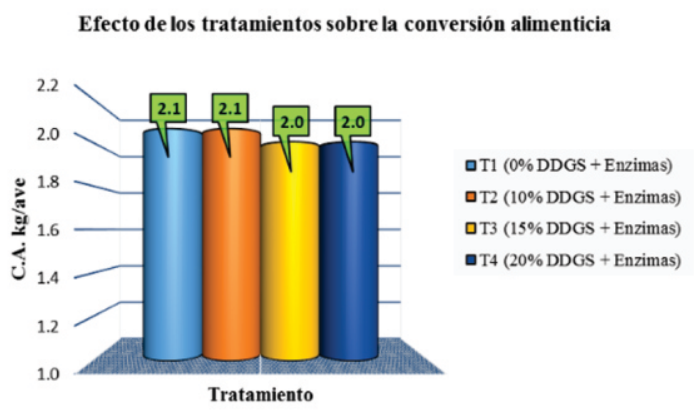

Figura 3. Se muestra los resultados de conversión alimenticia en gallinas Lohmann Brown Classic

En la figura 4 se presentan los resultados de pigmentación de yema de huevos en gallinas Lohmann Brown - Classic.

El T4 (20\% de DDGS + enzimas) mostró mayor pigmentación con 7.5, determinada con el Abanico de Roche.

Figura 4. Pigmentación de yema

Tabla 5. Comparación de Media Tukey sobre el consumo de materia seca

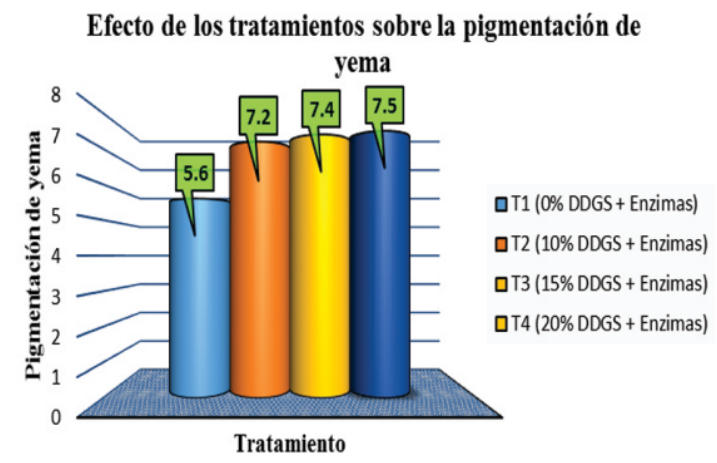

\begin{tabular}{|l|c|}
\hline Tratamiento & $\begin{array}{l}\text { Pigmentación de yema } \\
\text { (media) }\end{array}$ \\
\hline T4 & $7,5 \pm 0,07 \mathrm{a}$ \\
\hline T3 & $7,4 \pm 0,07 \mathrm{a}$ \\
\hline T2 & $7,2 \pm 0,07 \mathrm{a}$ \\
\hline T1 & $5,6 \pm 0,07 \mathrm{~b}$ \\
\hline
\end{tabular}

En la prueba de Tukey muestra que no existen diferencias significativas entre los T4, T3 y T2 pero si comparamos con el $\mathrm{T} 1$ observamos que existen diferencias significativas.

En la Figura 5 se muestra los pesos de huevos de gallinas tratadas con diferentes niveles de DDGS más enzimas en la dieta.

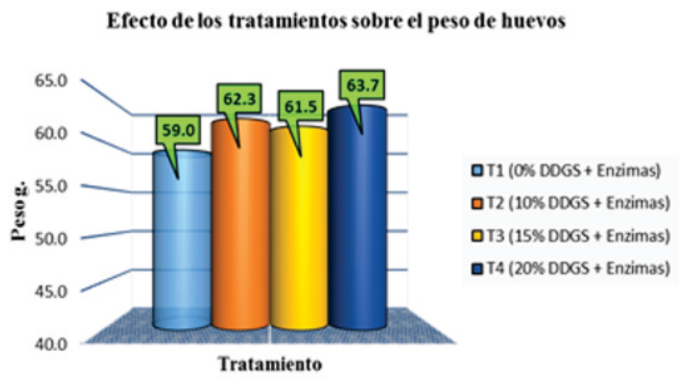

Figura 5. Peso del huevo para cada uno de los tratamientos

Se aprecia que el T4 obtuvo mejor resultado con respecto al peso del huevo con $63,7 \mathrm{~g}$, siendo el de menor valor el $\mathrm{T} 1 \mathrm{con} 59,0 \mathrm{~g}$.

Tabla 6. Comparación de Medias Tukey del peso de huevos

\begin{tabular}{|l|c|}
\hline Tratamiento & \multicolumn{2}{|c|}{$\begin{array}{l}\text { Peso del huevo (media } \\
\text { en } \mathrm{g})\end{array}$} \\
\hline T4 & $63,7 \pm 0,52 \mathrm{a}$ \\
\hline T3 & $62,3 \pm 0,52 \mathrm{ab}$ \\
\hline T2 & $61,5 \pm 0,52 \mathrm{~b}$ \\
\hline T1 & $59,0 \pm 0,52 \mathrm{c}$ \\
\hline
\end{tabular}

En la Tabla 6 se observa que el T4, T2 y T3, muestran diferencias significativas con respecto al $\mathrm{T} 1$, pero entre el T4 y T2 no presenta diferencias significativas.

En la Figura 6 se muestra los datos recolectados de una

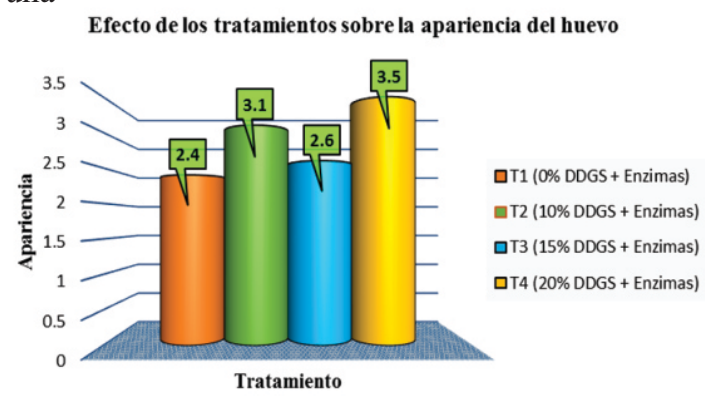

Figura 6. Apariencia del huevo de los tratamientos

El T4 (huevo con 20\% DDGS), se obtuvo el mejor puntaje de 3,7 en la evaluación de apariencia y el T1 (huevo con $0 \%$ DDGS) con menor puntaje de 2,4 .

Efecto de los tratamientos sobre el sabor del huevo

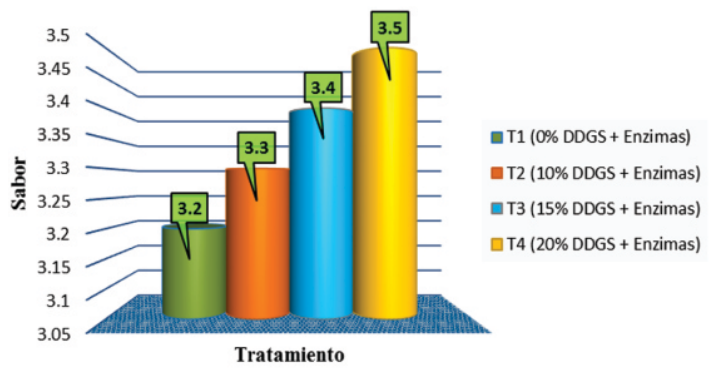

Figura 7. Sabor del huevo para los cuatro tratamientos 
El T4 (huevo con 20\% DDGS) es el de mayor calificación con 3,7 de puntaje y el T1 (huevo con $0 \%$ DDGS) con menor puntaje de 3,2 .

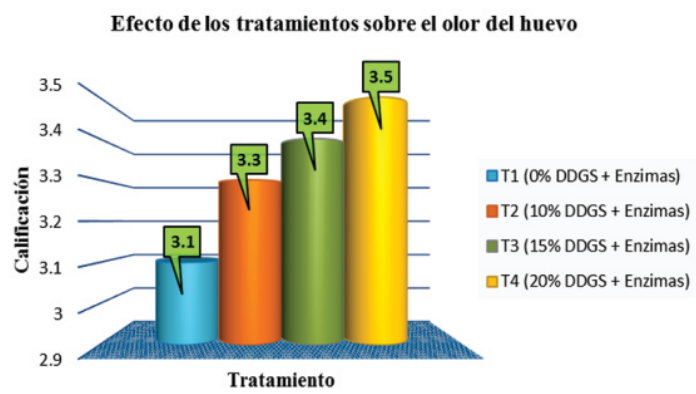

Figura 8. Olor del huevo de los tratamientos según la escala hedónica

El T4 (huevo con 20\% de DDGS) es el que mostró mayor puntaje con respecto al olor del huevo con 3,6 de puntaje y en el T1 (huevo sin DDGS) se obtuvo el menor puntaje de 3,1 .

En la tabla 7, Se observa que la mayor utilidad en el experimento se consiguió con el T3 (15\% DDGS), por cuanto se determinó un beneficio/costo de 0,003 soles por cada kilo de huevo producido.

Tabla 7. Comparación de rentabilidad de los tratamientos

\begin{tabular}{|c|c|c|c|c|}
\hline Descripción & T1 & $\mathbf{T 2}$ & T3 & T4 \\
\hline $\begin{array}{l}\text { Peso huevo } \\
\text { (kg) }\end{array}$ & 0,059 & 0,062 & 0,061 & 0,063 \\
\hline $\begin{array}{l}\text { Precio huevo/ } \\
\text { kg (S/.) }\end{array}$ & 2,90 & 3,40 & 3,80 & 3,60 \\
\hline $\begin{array}{l}\text { Postura } \\
\text { diaria }(\%)\end{array}$ & 73,30 & 86,30 & 95,20 & 91,60 \\
\hline $\begin{array}{l}\text { Ingreso bruto } \\
\text { diario por } \\
\text { huevo (S/.) }\end{array}$ & 0,14 & 0,19 & 0,22 & 0,21 \\
\hline $\begin{array}{l}\text { Consumo de } \\
\text { concentrado } \\
\text { (kg/ave) }\end{array}$ & 0,089 & 0,112 & 0,117 & 0,1114 \\
\hline $\begin{array}{l}\text { Precio } \\
\text { concentrado } \\
\text { por } \mathrm{kg}(\mathrm{S} / .)\end{array}$ & 1,62 & 1,61 & 1,60 & 1,59 \\
\hline Costo diario & 0,14 & 0,18 & 0,19 & 0,18 \\
\hline $\begin{array}{l}\text { Utilidad por } \\
\text { huevo } \\
\text { producido }\end{array}$ & 0,000 & 0,001 & 0,003 & 0,002 \\
\hline
\end{tabular}

\section{DISCUSIÓN}

Con una dieta de $15 \%$ de DDGS, se obtuvo el mayor porcentaje de postura $(95,2 \%)$, valor superior a los encontrado por Roberson (2005), en gallinas HY -LINE variedad Brown alimentadas con DDGS $(81,98 \%)$. El mismo autor, obtuvo de valores de alimento consumido de $113 \mathrm{~g} / \mathrm{ave} /$ día con una inclusión de $10 \%$ de DDGS, mientras que en esta investigación con $15 \%$ de inclusión, se obtuvo un promedio de $117,7 \mathrm{~g} / \mathrm{ave} / \mathrm{dí}$. Valores que se encuentran en el rango recomendado en la Guía de manejo de la ponedora Lohmann Brown - Classic (Lohmann, 2013), cuyos valores deben estar entre 110 y $120 \mathrm{~g} /$ ave/día.

Todos los tratamientos arrojaron valores de conversión alimenticia de acuerdo a lo refrenciado por Lohmann (2013), quienes refieren que los valores deben estar entre 2,0 y 2,2 $\mathrm{kg}$ de alimento para producir un $\mathrm{kg}$ de huevo.

La inclusión de 20\% de DDGS, mostró mejor respuesta estadística en la pigmentación de la yema de huevo (7,5), sin embargo, Matterson et al. (1996) obtuvieron valores de 8,22 son sólo $15 \%$ de DDGS; esto podría deberse a la composición de DDGS, por otro lado Zamora (2015), empleando harina de Leucaena leucocephala obtuvieron valores de pigmentación muy superiores (10).

A mayor incorporación de DDGS en la dieta de las gallinas, mayor incremento en el peso del huevo concordando con Lohmann (2013), quien indica que los resultados pueden variar, de acuerdo a las condiciones nutricionales, densidad, ambiente físico y biológico que se proporcionan a las aves.

\section{CONCLUSIONES}

Se mejoraron los parámetros como pigmentación de la yema del huevo, peso del huevo, consumo de alimento, porcentaje de postura, a medida que se incrementó los niveles de DDGS en la dieta.

La conversión alimenticia no varió de manera significativa a medida que se aumentó los niveles de DDGS en la dieta.

La adición de $15 \%$ de DDGS en dieta de gallinas ponedoras aumenta el beneficio económico en 0,03 más por kg de huevo producido comparado al testigo.

El DDGS es un alimento palatable ya que las gallinas Lohmann Brown - classic no tuvieron ningún inconveniente para consumirlo. Además el cambio de ración alimenticia de las aves en los tratamientos no afecto en el peso.

\section{REFERENCIAS BIBLIOGRÁFICAS}

Aparicio, A., Barroeta, A. C., Lopez-Sobaler, A. M., y Ortega, R. (2008). Tabla de Composición del Huevo de Gallina. Instituto de Estudios del Huevo. Madrid, España. 
Recuperado de www.institutobuevo.com

Blas, C. D., Mateos, G., y Rebollar, P. (2007). DDGS de maíz (granos de destilería, DDG, y solubles, DDS). Universidad Politécnica de Madrid, España.

Matterson, L. D., Tlustohowicz, J., and Singsen, E. P. (1966). Corn Distillers Dried Grains with Solubles in Rations for High-Producing Hens 1 2. Poultry Science, 45(1), 147151.

Paucar, H. G. (2016). Caracterización de mercado del huevo comercial (Gallina Lohmann Brown) versus el huevo criollo (Gallina de campo) en la provincia de Chimborazo (Bachelor's thesis, Escuela Superior Politécnica de Chimborazo).

Roberson, K., Kalbfleisch, J., Pan W., and Charbeneau, R. (2005). Effect of corn distiller's dried grains with solubles at various levels on performance of laying hens and yolk color. Intl J. Poultry Sci. $4(2): 44-51$.

Spiehs, M. J., Whitney, M. H., and Shurson, G. C. (2002). Nutrient database for distiller's dried grains with solubles produced from new ethanol plants in Minnesota and South Dakota. Journal of animal science, 80(10), 2639-2645.

Zamora, S. J. (2015). Efecto de harina de leucaena (Leucaena leucocephala) en la calidad y producción de huevos en gallinas ponedoras Hisex Brown. Revista Cientifica UNTRM: Ciencias Naturales e Ingeniería, 1(1), 33-37. 